Exploring Prompt Measurement Methods for $(n, 2 n)$ Cross Sections on Radioactive Targets

L. Ahle

January 9, 2006 
This document was prepared as an account of work sponsored by an agency of the United States Government. Neither the United States Government nor the University of California nor any of their employees, makes any warranty, express or implied, or assumes any legal liability or responsibility for the accuracy, completeness, or usefulness of any information, apparatus, product, or process disclosed, or represents that its use would not infringe privately owned rights. Reference herein to any specific commercial product, process, or service by trade name, trademark, manufacturer, or otherwise, does not necessarily constitute or imply its endorsement, recommendation, or favoring by the United States Government or the University of California. The views and opinions of authors expressed herein do not necessarily state or reflect those of the United States Government or the University of California, and shall not be used for advertising or product endorsement purposes.

This work was performed under the auspices of the U.S. Department of Energy by University of California, Lawrence Livermore National Laboratory under Contract W-7405-Eng-48. 


\title{
Exploring Prompt Measurement Methods for (n,2n) Cross Sections on Radioactive Targets
}

\author{
Larry Ahle - Lawrence Livermore National Laboratory \\ January 5, 2005
}

\section{Executive Summary}

This report summarizes a study of possible neutron detection technologies for performing prompt $(n, 2 n)$ measurements on radioactive targets of the type that could be made at the Rare Isotope Accelerator (RIA). The report recommends conducting further research on high-pressure ${ }^{3} \mathrm{He}$ gas scintillators as it is the best candidate technology. These detectors meet the requirements of a fast response time (fall times around 5-10 ns), gamma ray suppression, (all gamma rays below about $900 \mathrm{keV}$ can be easily discriminated against), and can be easily configured into a $4 \pi$ array. The one requirement that these detectors fall short is efficiency, but less than a factor of 10 improvement is needed. The possibility of pulse shape discrimination should also be explored for these detectors as this would help to distinguish gamma rays above $900 \mathrm{keV}$ from neutrons. In addition to R\&D work on these detectors, Monte Carlo simulations and target development are also recommended areas of further study.

\section{Introduction}

With the advent of high power radioactive ion beam facilities, the possibility exist for creating targets of short lived isotopes and performing neutron cross section measurements. Of particular interest to stockpile stewardship are $(n, 2 n)$ cross sections on proton rich isotopes near stability. While many of these $(n, 2 n)$ measurements can be carried out using the activation technique, many can not because the half life of the reaction product is too short or the decay signal of the reaction product can not be seen above the large background of the target itself. Additionally, the activation technique requires a very pure target, usually free of the final reaction product at the 1 part in $10^{9}$. Many of these problems could be overcome if the measurement was done promptly, identifying $(n, 2 n)$ events as they happen. While gamma rays have been used to determine $(n, 2 n)$ events [1], this is impractical for radioactive targets because of the high gamma ray background of the target. Detecting both neutrons is another technique that has been used in the past [2] and has the potential to work for radioactive targets, though it is not clear what type of neutron detector should be used. While many types of neutron detectors exist, this application requires neutron detectors that are fast, relatively insensitive to gamma rays, and detect neutron with energies from several hundred keV to a few $\mathrm{MeV}$. This report starts to address this issue by reporting on a survey of neutron detection technology and making recommendations as to which if any should be pursued further. 


\section{Requirements}

In order to make an assessment of the applicability of various neutron detection technologies, one first must define the environment in which the detector must operate. To start with, it is assumed these measurements would be performed at RIA using the targets that could be made at such a facility. This implies targets of $10^{16}$ atoms and activities as much as a few Curies for nuclei with a one day half-life. It is also assumed that when the target atoms decay, they emit gamma rays at $500 \mathrm{keV}$ and nearly $100 \%$ of the time. Obviously, not all targets will emit such radiation but it provides a reasonable upper limit for this study. Additionally it is assumed the target is irradiated with a well collimated neutron beam with a flux of $10^{9} \mathrm{n} / \mathrm{cm}^{2} / \mathrm{s}$ on target. This is by no means an easy accomplishment, but how to meet this goal is outside the scope of this report. It is with these assumptions that the rest of the analysis on requirements will follow.

\section{The Good - The Signal}
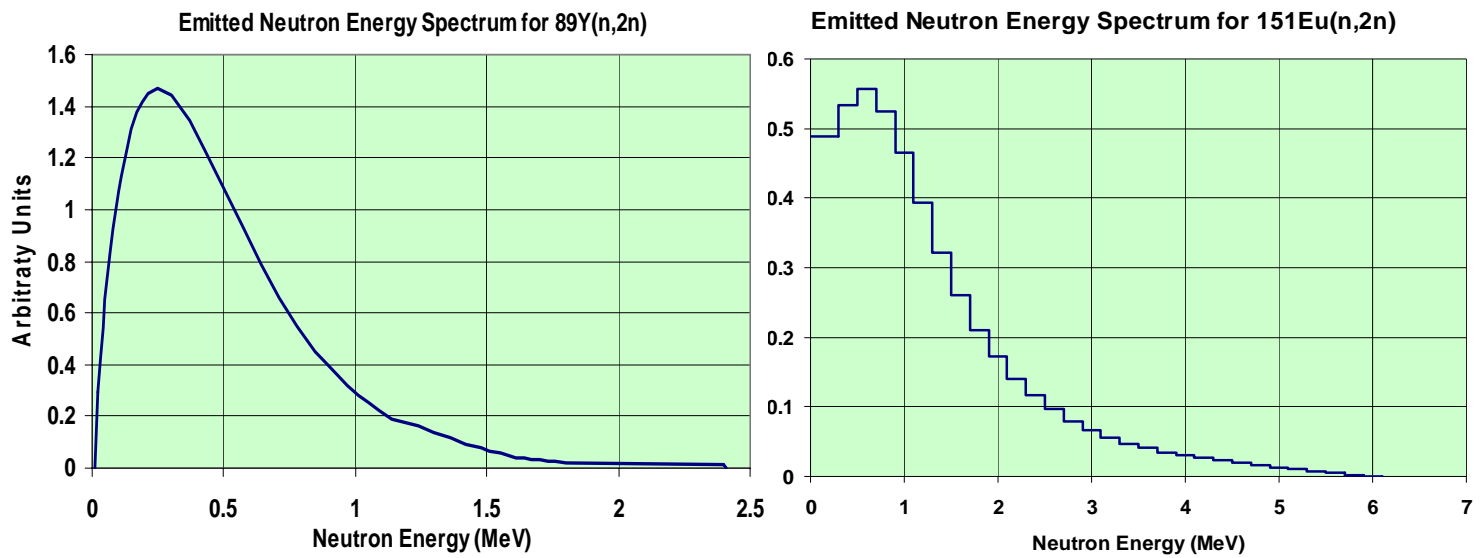

Figure 1: Plots of emitted neutron energy spectra for $(n, 2 n)$ reactions at incident neutron energy of $14 \mathrm{MeV}$ as calculated by ENDF-BVI. The plot on the left is for ${ }^{89} \mathrm{Y}(\mathrm{n}, 2 \mathrm{n})$ and the plot on the right is for ${ }^{151} \mathrm{Eu}(\mathrm{n}, 2 \mathrm{n})$.

Given $10^{16}$ atoms, a neutron flux of $10^{9} \mathrm{n} / \mathrm{cm}^{2} / \mathrm{s}$, a cross section or 0.5 barns, and one day irradiation (limited by half-life of target and/or product), then a total $4 \times 10^{5}$ (n,2n) events will occur during the experiment with a peak rate of 5 events a second. In most cases a $5 \%$ accurate measurement would be required and there will almost certainly be a need for background subtraction, increasing the error of the measurement. Thus, the detector system should be efficient as possible for detecting $(n, 2 n)$ events, with a lower limit of $1 \%$. At the neutron energies of interest, around $14 \mathrm{MeV}$, all angles are kinematically possible so that the detector system must cover all of $4 \pi$ to maximize overall efficiency. The energy range of these evaporated neutrons depends somewhat on the nucleus but most of the of the neutron fall 0.1-3 MeV. Figure 1 plots the ENDF-BVI evaluation for the neutron energy spectrum for the reaction ${ }^{89} \mathrm{Y}(\mathrm{n}, 2 \mathrm{n})$ and ${ }^{151} \mathrm{Eu}(\mathrm{n}, 2 \mathrm{n})$. Furthermore, there is only a weak correlation in angle and energy between the two emitted neutrons, requiring both neutrons to be detected. Thus, what ever neutron detection technology is chosen, it must provide a single neutron detection efficiency of $10 \%$ for energies from 0.1-3 MeV and must lend itself to $4 \pi$ angular coverage. 


\section{The Bad - The Gamma Background}

Given the radioactive target could be as much as a Curie or more of activity, the detector system must be able to handle a high gamma ray background that is uniform in space and time. Clearly a fast detector would be desirable over a slow one, but with one Curie corresponding to 37 decays per nanosecond, speed is not enough to reduce the background to acceptable levels. Given the range of neutron energies and a $50 \mathrm{~cm}$ path length from target to detector, then largest possible timing difference between two coincident neutrons is 94 ns. Thus, even an infinitely fast detector system will still have to handle the gamma rays from 3700 decays. Segmenting the $4 \pi$ detector will help as each detector element will see only part of the 3700 decays. But even with 1000 modules and a $10 \%$ efficiency for detecting gamma rays, over one third of the detector elements will still record a signal from gammas in any given 100 ns time window. One could try to reduce the efficiency for detecting gammas, but in general reducing gamma ray detection efficiency also reduces neutron detection efficiency for a given system. Thus, speed and segmentation together will still probably not be enough to handle this background. Fortunately, many neutron detectors also offer some method for distinguishing gamma ray events from neutron events, either by pulse shape discrimination or signal strength. Each detector technology will have to be looked at in a case by case basis to determine if its method of discrimination will work in this environment. In any event, this $4 \pi$ neutron detector system must also be divided into many detector elements, each with a signal decay time of tens of nanoseconds or less and a method for discriminating between neutrons and gammas.

\section{The Ugly - The Scattered Neutron Background}

Given the small amount of target atoms, the targets for irradiation will include a backing material with several orders of magnitude more atoms within the neutron beam spot. The largest impact on the surrounding neutron detectors will be elastically scattered neutrons. Assuming a $100 \mu \mathrm{g} / \mathrm{cm}^{2}$ carbon backing then about 5 times a second there will be two elastically scattered neutrons within 100 ns of each other. Thus, the detector system must not only distinguish gamma rays from neutrons, but elastic scattered neutrons from the evaporated neutrons. Given the larger atomic mass of the backing material, the elastically scatter neutrons will be somewhat isotropic and the energy of the neutron will be still be near the neutron beam energy. For example, the lowest possible energy for 14 $\mathrm{MeV}$ neutrons scattered off ${ }^{12} \mathrm{C}$ is about $10 \mathrm{MeV}$. Thus, these two sets of neutrons can be distinguished by there energy. This can be done by the signal produced by the neutron detector or by time of flight. There would be at least a 10 ns separation in arrival times assuming the $50 \mathrm{~cm}$ path length from target to detector. Of course to take advantage of the time of flight difference, the neutron beam would need to be pulsed, thus, resulting in a lower neutron flux.

\section{The Really Ugly - Background (n,2n) Events}

One background component which will be indistinguishable from real events are $(n, 2 n)$ reactions on background material. The neutrons will have essentially the same characteristics as the reaction product neutrons including the same timing. Thus, the only way to deal with this background is reduction and subtraction. Two key items for 
reduction will be the appropriate choice of target backing material and a vacuum line for the neutron beam.

Given the production rates of radioactive isotopes even at a facility like RIA, there will be only $10^{16-17}$ atoms of target material (about 1-10 $\mu$ g). This implies a backing material is needed, which will be $10^{19-20}$ atoms $/ \mathrm{cm}^{2}$ of material. Given a $1 \mathrm{~cm}$ diameter collimated neutron beam $\left(0.78 \mathrm{~cm}^{2}\right)$, then the amount backing material intercepted by the beam will be as much 4 orders of magnitude more than the target material. This would be prohibitive unless the threshold for $(\mathrm{n}, 2 \mathrm{n})$ reactions on the backing material were sufficiently high. Fortunately ${ }^{12} \mathrm{C}$ has such a high threshold, $20.3 \mathrm{MeV}$, thus making carbon backed targets a possibility. However, the backing material needs to be pure ${ }^{12} \mathrm{C}$, as ${ }^{13} \mathrm{C}$ has a $(\mathrm{n}, 2 \mathrm{n})$ threshold of only $5.3 \mathrm{MeV}$. It should also be possible to make oxides target material as ${ }^{16} \mathrm{O}(\mathrm{n}, 2 \mathrm{n})$ has a threshold of $16.7 \mathrm{MeV}$. Both ${ }^{17} \mathrm{O}$ and ${ }^{18} \mathrm{O}$ have a low threshold, so there can't be too much extra oxygen in the target. Some amount of hydrogen in the target is also possible, since $(n, 2 n)$ reactions cannot occur. However, neutron scattering off hydrogen does produce neutrons in the evaporated neutron energy range at angles just forward of ninety degrees.

${ }^{14} \mathrm{~N}(\mathrm{n}, 2 \mathrm{n})$ has a threshold of $11.31 \mathrm{MeV}$. Since nitrogen is the largest component of air, neutron reactions in air would also contribute to this background. If we have a spherical array of 1 meter inner diameter, the neutron beam will see a target of $3 \times 10^{21}$ nitrogen atoms from one end of the array to the other. Reactions on these nitrogen atoms would overwhelm any signal from the target. This background component can be eliminated if there is a vacuum beam pipe traversing the array. The vacuum need not be connected to same vacuum as the beam that creates the neutron, but the end flanges for the neutron vacuum line should be some distance from the array to allow for shielding. The diameter of the vacuum line will have to be optimized by trading off scattering and background issues versus geometrical acceptance of the array.

In terms of the choice of neutron detector, the less material the detector system adds to the area surrounding the target the better, especially for high Z material. Even if time of flight is used and these background events fall outside of the time window, one will still need to wait for these background events to subside before the next beam pulse. Thus, the more material, the longer the wait time will be and the lower the average neutron flux on target.

\section{Survey of Neutron Detection Technology}

From the previous section, the ideal neutron detection technology must be able to detect neutrons from 0.1-5 MeV with at least $10 \%$ efficiency, have decay times of tens of nanoseconds or less, be able to distinguish $14 \mathrm{MeV}$ neutrons from low energy neutrons, and have no response to gamma rays. It must also be readily configured into an array to cover $4 \pi$. Given the energy range of interest, most neutron detectors rely on one of three basic detection principles, moderation, proton recoil, and direct nuclear reaction. These three concepts are discussed below in context of this application. 


\section{Moderation}

Many neutron detectors rely on moderating high energy neutron to lower energies and use large reaction cross sections at low energies to detect neutrons. These large cross sections combined with the appropriate geometry of the moderator can lead to very high efficiencies, as high as $80-90 \%$. Also, the neutron detection reaction usually releases several $\mathrm{MeV}$ of energy in charged particle or gammas, making a signal that is easily distinguished from single background gamma rays. The price for this is the loss of neutron energy information and the long time window needed for coincident neutrons.

Since the nuclear reaction that produces the detected signal takes place at nearly the same energy regardless of the initial energy of the neutron, there is no information in the signal with regards to neutron energy. Additionally, time of flight can not be used given the timing variance of the signal. The moderation of a neutron from several $\mathrm{MeV}$ to near thermal energies, usually takes about 100 ns, but the time it takes to for the moderated neutron to induce the desired reaction is typically much longer. For Frehaut's experiments the capture time was 30-50 microseconds with a FWHM of 20 microseconds. With proper choice of materials and size of detector, these times can be reduced, but the reduction of the variance in the capture time of several nanoseconds is not achievable. A much larger flight path would reduce the needed time resolution, but this would be impractical because of the required $4 \pi$ angular coverage. Thus it is not possible to determine energy from time of flight.

The inability to distinguish $14 \mathrm{MeV}$ neutrons from lower energy neutrons and the long time gate for coincident neutrons conspire to create a large background for this type of detector. The rate of detected $(n, 2 n)$ events is,

$$
R_{(n, 2 n)}=\sigma_{T} T_{T} n \varepsilon_{2 n}^{2}
$$

where $\sigma_{T}$ is the cross section for the desired reaction $\left(5 \times 10^{-25} \mathrm{~cm}^{2}\right), T_{T}$ is the number of target atoms $\left(10^{16}\right), n$ is the neutron flux $\left(10^{9} \mathrm{n} / \mathrm{cm}^{2} / \mathrm{s}\right)$, and $\varepsilon_{2 n}$ is the efficiency for detecting a single evaporated neutron (80\%). The rate for detecting two scattered 14 $\mathrm{MeV}$ neutrons in a time window $\Delta t$ is

$$
R_{2(n, n)}=2 R_{(n, n)}^{2} \Delta t=2\left(\sigma_{B} T_{B} n \varepsilon_{n}\right)^{2} \Delta t
$$

where $\mathrm{R}_{(\mathrm{n}, \mathrm{n})}$ is the rate for detecting a single scattered neutron, $\sigma_{T}$ is the cross section for for neutron scattering of the backing material $\left(1 \times 10^{-24} \mathrm{~cm}^{2}\right), T_{T}$ is the number of backing material atoms in the cross sectional area of the neutron beam $\left(2.5 \times 10^{19}\right)$, and $\varepsilon_{n}$ is the efficiency for detecting a single scattering neutron (80\%). Using the numbers listed above, then the ratio of coincident scattered neutrons in a time window of 10 microseconds compared to $(n, 2 n)$ events is over 100 . In general, the efficiency for a scattered $14 \mathrm{MeV}$ neutron would be less than an evaporated neutron, but only slightly. This analysis also ignores the impact of detected events which have one $(n, 2 n)$ neutron and one scattered neutron.

\section{Proton Recoil}

Another common approach for detecting high energy neutrons is proton recoil. This usually involves an organic scintillator of some type that is high in hydrogen content. A 
neutron elastically scatters off a proton, transferring energy to the proton, which then releases its energy to the medium, some of which ends up as scintillation light. Another variant on this approach is to use a thin piece of plastic with a particle detector nearby. Given the limitation on the thickness of the plastic to allow the proton to escape, the efficiency for detecting the neutron is quite low and inadequate for this application. The scintillator can achieve the desired efficiency by choosing the appropriate thickness, around $0.5 \mathrm{~cm}$ for $1 \mathrm{MeV}$ neutrons. Additionally one can use pulse shape discrimination to distinguish between gamma rays and neutrons. Also, these scintillators are usually quite fast, allowing from time measurements to determine neutron energy. Unfortunately, there are other issues that make proton recoil far from the perfect choice.

First, organic scintillators are sensitive to gamma rays with an expected detection efficiency of $10 \%$. Thus, even with a thousand modules in the detector system, roughly one third of those detectors in 100 ns time window will indicate a response due to the gammas implying a third of the detector system blind to neutrons. This would result in an order of magnitude loss in efficiency for $(n, 2 n)$ events. While, it is possible to discriminate photons from protons in these detectors, it is extremely difficult to do this if the module was hit by both a photon and a neutron.

Second, even if the gamma ray background issue is solved, organic scintillators tend to have different response functions to different species of particles. Thus, $1 \mathrm{MeV}$ proton does not produce the same amount of scintillation light as a $1 \mathrm{MeV}$ electron, with the proton producing less light. This effect has been studied for many different types of organic scintillators, and empirical relationships between electron energy and proton energy have been determined [3]. For stilbene detectors, an organic crystal, this relations between electron and proton energy has been determined to be $E_{e}=0.15^{*} E_{p}{ }^{1.45}$. Thus, a one $\mathrm{MeV}$ proton produces the same amount of light as a $150 \mathrm{keV}$ electron. For some organic liquids the number is closer to $300 \mathrm{keV}$. Thus, neutrons in the several hundred $\mathrm{keV}$ range are very difficult to detect, especially in high gamma background environments. Additionally, it is harder to discriminate between gammas and neutrons at these low energies.

And third, even if the challenges of detection of low energy neutrons are overcome, the proton can recoil with a range of energies. In fact, all recoil proton energies, between 0 and the neutron energy are equally likely. This makes the interpretation of the measured spectra even more challenging. For instance, $21.5 \%$ of the proton recoils from a $14 \mathrm{MeV}$ neutron will be below $3 \mathrm{MeV}$, the desired range for $(\mathrm{n}, 2 \mathrm{n})$ neutrons. Also, if one set a detector cut of $500 \mathrm{keV}$ of proton recoil energy ( $<100 \mathrm{keV}$ of electron equivalent energy), than the best possible detection efficiency of $600 \mathrm{keV}$ neutrons is $17 \%$ while it is $50 \%$ for one $\mathrm{MeV}$ neutrons. While a careful analysis maybe able to interpret the data properly with the use of time of flight to reject scattered neutrons, the several hundred keV range of neutron energy will be the hardest region to interpret and have the largest efficiency corrections. It is this very region that will have the most number of neutrons from $(n, 2 n)$ events. 


\section{Nuclear Reactions}

Obviously, all neutron detection methods rely on neutron reactions to produce a detectable signal, but this section discusses using inelastic nuclear reactions at the incident neutron energy. Many different reactions have been tried for various applications and neutron energies some of which are not appropriate for this application. For instance, there has been recent work done using the ${ }^{12} \mathrm{C}(\mathrm{n}, \alpha)$ reaction to detect 14 $\mathrm{MeV}$ neutrons [4]. Unfortunately, this reaction has a threshold of $6.18 \mathrm{MeV}$ and thus could not be used in this application. Three neutron reactions, $(\mathrm{n}, \mathrm{f}),{ }^{6} \mathrm{Li}(\mathrm{n}, \mathrm{a})$, and ${ }^{3} \mathrm{He}(\mathrm{n}, \mathrm{p})$, are discussed below in some detail.

Neutron detectors that rely on fission have been used extensively in many applications. Most of these detectors have a thin actinide layer such as ${ }^{235} \mathrm{U}$ or ${ }^{238} \mathrm{U}$, surrounded by a gas volume for detecting the high energy fission fragments produced in the reaction. Since the fragments have very high energy, they produced a unique signal that can be easily seen above any gamma, or charged particle background. Since this energy is much higher than the incident neutron energy, no neutron energy information is present in the signal, though these detectors can be made fast enough to allow time of flight information to be used. Unfortunately, the required thickness to allow the fission fragments to escape results in a very low efficiency for detecting neutrons. One could imagine stacking many fission neutron detectors together to increase efficiency, but it would take hundreds of detector to approach a $10 \%$ efficient detector. And if one built an array of these stacks, there would also be the issue of neutrons emitted from the fission reaction creating other fission reactions in nearby detectors. Additionally care must be taken into the choice actinide used. ${ }^{238} \mathrm{U}$ cannot be used because the neutron energy threshold of (n,fission) is too high, around $1 \mathrm{MeV}$. Similarly ${ }^{235} \mathrm{U}$ and ${ }^{239} \mathrm{Pu}$ are not ideal because of the large (n,fission) cross sections for thermal and epithermal neutrons. In principle, these can be shielded against, though it would be more difficult to shield against low energy neutrons emitted from fission reactions in the detector themselves then from general room background. One actinide whose (n,fission) cross section is much more suitable to this application would ${ }^{237} \mathrm{~Np}$, which has been used in neutron detectors. An extensive search for other possible actinides, however, has not been done in part due to efficiency issues raised above.

The ${ }^{6} \mathrm{Li}(\mathrm{n}, \alpha)^{3} \mathrm{H}$ reaction, which has a $\mathrm{Q}$-value of $4.78 \mathrm{MeV}$, can also be used to detect neutrons. In most cases, ${ }^{6} \mathrm{Li}$ is added to glass scintillators to convert the kinetic energy of the charged particle products to light. Since the neutron detection relies on scintillation, these detectors are also sensitive to gamma rays. The glass scintillators also suffer from the same phenomena of unequal light output for different particles. Thus, reaction production of $4.78 \mathrm{MeV}$ produce about the same light output as $1.2 \mathrm{MeV}$ gamma rays [5]. These detectors are fast and have been used for time of flight measurements to determine neutron energy. The cross section for this reaction in the energy range of interest is not very constant, however. There is a resonance peak near $250 \mathrm{keV}$ and the cross section starts to rise again at $3 \mathrm{MeV}$. The ratio of the highest to lowest cross section value in the neutron energy range from $200 \mathrm{keV}$ to $3 \mathrm{MeV}$ is over a factor of 10 . Above neutron energies of $2.5 \mathrm{MeV}$ the dominant neutron reactions becomes ${ }^{6} \mathrm{Li}\left(\mathrm{n}, \mathrm{n}{ }^{\prime} \mathrm{d}\right)^{4} \mathrm{He}$. Since this is a three body reaction and not all of the output products are charged, this reaction will 
produce a continuum background on the resultant energy spectrum. While most of the evaporated neutrons are below $2.5 \mathrm{MeV}$, the $14 \mathrm{MeV}$ scattered neutrons are not. Given the abundance of these neutrons, this will probably result in a significant background contribution. Additionally, given the concentration of ${ }^{6} \mathrm{Li}$ in theses scintillators, about $7 \%$ by weight, the glass would need to be approximately $35 \mathrm{~cm}$ thick to achieve the desired efficiency.

Another reaction commonly used to detect high energy neutrons is ${ }^{3} \mathrm{He}(\mathrm{n}, \mathrm{p})$. This reaction results in two charged particles with a total positive Q-value of $0.764 \mathrm{MeV}$, making it easy to distinguish neutrons from low energy gamma rays. Also the cross section is fairly constant over the range of interest at about 1.0 barns. The ratio of the highest to lowest cross section value for neutron energies between $200 \mathrm{keV}$ and $3 \mathrm{MeV}$ is less than a factor of two. Additionally, the cross section falls significantly for higher energy neutrons, approaching 100 mbarns for $14 \mathrm{MeV}$ neutrons. While there is no competing nuclear reaction for higher energies, elastic collisions producing recoil ${ }^{3} \mathrm{He}$ would also produce a detectable signal. These reactions will complicate separation of 14 $\mathrm{MeV}$ scattered neutrons without using time of flight. Since ${ }^{3} \mathrm{He}$ is a gas, this detection scheme is most often used with ionization chambers or proportional counters. These detectors however, are generally quite slow with rise times on the order of 1 microsecond. Another detector variant uses ${ }^{3} \mathrm{He}$ as a noble gas scintillator. In this scheme, ${ }^{3} \mathrm{He}$ is the target for neutron reactions and the scintillation medium. Often, other gases, such as Xenon, are added in small quantities to increase light output. Scintillators of this type offer two advantages over the scintillators discussed earlier. First, noble gas scintillators are very fast with decay times of around a few nanoseconds. Second, the light output only depends on the energy of the particle, not on its species [6]. Thus, the $0.764 \mathrm{MeV}$ q-value implies that gamma rays below $0.9 \mathrm{MeV}$ are easily discriminated against neutrons, which is not too far different from the lithium glass scintillators. The critical issues for this reaction is getting the efficiency high enough and designing a system to cover $4 \pi$.

\section{Examples}

From table 1 , the ${ }^{3} \mathrm{He}$ gas scintillators appears to be the most promising choice for neutron detection technology, but issues of efficiency and a design of a $4 \pi$ system remain. This section will look at those issues in more detail. Additionally, the gadolinium loaded, liquid scintillator ball is also looked at some detail as it this system that was used for many $(n, 2 n)$ measurements on stable targets. While it is natural to first try to extend this system to this environment, there are enough differences in the measurement environment that make it questionable as to whether this is the best approach.

\section{Gadolinium-loaded Liquid Scintillator Balls}

As has been mentioned before, gadolinium loaded scintillator balls have used to measure $(\mathrm{n}, 2 \mathrm{n})$ directly for stable targets and some actinides. However, this detector relies on moderation and as such all the disadvantages of such detectors described above holds. The biggest issue is the background from the scattered neutrons off the backing material. From the analysis above, the background can be reduced by lowering the neutron flux, 
decreasing the time window for coincidence, or reducing the efficiency for detecting a scattered neutron relative to an evaporated neutron. Lowering the neutron flux, will limit the number of reactions that can be performed, while lowering the time window will be difficult since most of the time variance occurs while the neutron is at low energy not during the moderation process. While a thinner scintillator will reduce the efficiency for scatter neutrons more than evaporation neutrons a factor of 10 improvement will still leave the background as large as the real signal.

\begin{tabular}{|c|c|c|c|c|c|c|}
\hline Detector & TOF & $\begin{array}{c}\text { Energy } \\
\text { Range }\end{array}$ & $\begin{array}{c}\text { Neutron } \\
\text { Gamma } \\
\text { Separation }\end{array}$ & $\begin{array}{c}\text { Neutron } \\
\text { Energy } \\
\text { Dependent } \\
\text { Signal }\end{array}$ & Efficiency & $\begin{array}{c}4 \pi \\
\text { array }\end{array}$ \\
\hline Moderator & no & yes & yes & no & yes & yes \\
\hline Proton Recoil & yes & no & $\begin{array}{c}\text { Above } 1 \\
\text { MeV neutron } \\
\text { energy }\end{array}$ & $\begin{array}{c}\text { Yes but not } \\
\text { unique }\end{array}$ & yes & yes \\
\hline Fission & yes & marginal & yes & no & no & no \\
\hline${ }^{6} \mathrm{Li}(\mathrm{n}, \alpha)$ & yes & marginal & difficult & yes & no & yes \\
\hline $\begin{array}{c}{ }^{3} \mathrm{He}(\mathrm{n}, \mathrm{p}) \\
\text { gas scintillator }\end{array}$ & yes & yes & $\begin{array}{c}\text { Below 0.9 } \\
\text { MeV gamma } \\
\text { ray energy }\end{array}$ & yes & ???? & ???? \\
\hline
\end{tabular}

Table 1: Summary table of neutron detector technology and requirements

In principle, the signals from the moderation process should be present as the neutron loses most of its energy through proton scatter in the scintillator. Measuring and interpreting these signals would allow one to distinguish $14 \mathrm{MeV}$ neutrons from lower energy neutrons. Of course, to do this one must work with the same issues as the proton recoils detectors described above, as this is exactly the same process. The mixing of these signals with the gamma ray background would make them difficult to disentangle. In 10 microseconds, the decaying target itself would produce on average 40 detected gammas per detector in a 1000 element array. While the signals from the moderation occur in just a 100 ns time window, identify this $100 \mathrm{~ns}$ time window relative to a capture event will not be easy in this gamma ray environment.

\section{${ }^{3} \mathrm{He}$ Gas Scintillators}

The major issue confronting using the ${ }^{3} \mathrm{He}(\mathrm{n}, \mathrm{p})$ reaction as the neutron detector technology is designing a detector that has the appropriate efficiency. For a $10 \mathrm{~cm}$ thick detector volume, one would need to design a chamber to withstand approximately 400 atmospheres of pressure in order to achieve. For unrelated reasons, Los Alamos (A. E. Evans) looked into such a device in the early 1980's [7]. They designed a gas chamber that is roughly a cylinder $5 \mathrm{~cm}$ in diameter and $10 \mathrm{~cm}$ long with a phototube coupled to one end via a tempered glass end cap. The chamber was designed to accommodate a pressure of 150 atmospheres and as such its efficiency is approaching the desired efficiency. An efficiency of $1.3 \%$ for $500 \mathrm{keV}$ neutrons is reported, but this efficiency is reduced somewhat because to details in geometry which results in a detector volume 
significantly shorter than $10 \mathrm{~cm}$. In any advent, to reach the desired $10 \%$ efficiency, one only needs to extend on this work by a factor few and not orders of magnitude. Additionally, Evans' design should allow a close pack $4 \pi$ array by making only slight design modifications.

Thus it seems ${ }^{3} \mathrm{He}$ gas scintillators seem to be able to meet, or at least the possibility to meet, all of the desired criteria. It should be possible to design a system with $10 \%$ efficiency that is relatively constant between $200 \mathrm{keV}$ to $3 \mathrm{MeV}$ and be configured into a $4 \pi$ array. Because of the positive Q-value a light output that is independent of particle species, gamma rays below $900 \mathrm{keV}$ are easily ignored. For targets which emit higher energy gammas care would have taken in the analysis, though the efficiency for gamma in general will be low because of the small number electrons per helium atom. Also, it is not clear if pulse-shape discrimination could be used. It can be for most types of scintillators, but most scintillators' light output depend on particle species. By using time of flight, one can easily distinguished between the $14 \mathrm{MeV}$ scattered neutrons. If time of flight is not used, these neutron can be distinguished easily if ${ }^{3} \mathrm{He}(\mathrm{n}, \mathrm{p})$ reactions occur, but elastic reactions on the ${ }^{3} \mathrm{He}$ nuclei will produce signals that result in a significant background. It is not clear whether this background can be dealt with.

\section{The Next Steps}

From the above analysis, it is recommends that high pressure ${ }^{3} \mathrm{He}$ scintillators be pursued as the neutron technology for prompt (n,2n) measurements on radioactive targets. There are still several outstanding questions that require research and experimentation before a definitive decision on this technology can be made. The three major areas of $R \& D$ that should be addressed as the next steps are prototyping and testing, monte carlo simulation, and target development.

Prototyping and testing of these detectors is the step that needs to be done early on in the process. One must design a system that meets the desired efficiency and these types of detectors have not been testing in high gamma ray environments. Issues of maximizing light collection efficiency and energy resolution are always challenges for any detector. Also, the uniformity of the efficiency across the face and depth of the detector also needs to be understood. Gas purity will also be an issues and needs to be addressed. As mentioned earlier adding small amount of xenon increase light output, but other impurities such as oxygen and nitrogen can kill the light output. All of this will have to be studied in order optimized the detector design.

Monte Carlo simulations will also play a key role in the design of the final detector system. All the background estimates listed in this paper were estimated by hand calculations and ignore contributions from back scattered neutrons. Of course a lot of this contribution will depend on details of the "well-collimated" neutron beam and the neutron beam stop. But still very useful information can be obtained by performing a Monte Carlo simulation with very simple assumptions for these features. Though by using time of flight 
A critical factor in being able perform these is construction of the target. Unlike stable target experiments, pure targets will not be possible because of the small amount of material that is available. Even for stable targets, where much larger amounts are available, chemical properties often prohibit the formation of a pure target. These chemical issues, which vary from target to target, will also be present in making these radioactive targets. As mentioned above, the need for a backing material will cause two background components, scattered $14 \mathrm{MeV}$ neutrons and background (n,2n) reactions. While the scattered $14 \mathrm{MeV}$ background can be dealt with via the neutron detector and detection technique, the background $(n, 2 n)$ cannot. Thus, reducing this background is crucial, which can only be done be appropriate choice of backing material and control of impurities. While this issues cuts across all neutron detector technologies, its solution will depend on each individual target species. The chemical process for purifying the target material and than applying it to the backing material will be different for each chemical element. Nevertheless, providing a few proof in principle targets would go a long way to establishing the validity of these measurements.

\section{Summary}

This study had identified as high pressure ${ }^{3} \mathrm{He}$ gas scintillators as the most promising technology for performing $(\mathrm{n}, 2 \mathrm{n})$ cross section measurements on radioactive targets. This technology comes the closest to meeting the requirements of a fast response, gamma ray rejection, neutron energy range, and efficiency. Of these, the efficiency is the requirement that will be the hardest to meet for this technology. More detailed study and prototyping of these detectors need to be performed before a more definitive statement can be made as to the applicability of this technology. Additionally, Monte Carlo studies of theses measurements would also be useful in further developing these measurements. Research into target formation would also be useful and this directly impacts the background seen by the neutron detectors. Target issues also cut across all type of neutron detection technology. In short, there is a path forward, but much work remains before there is a viable method for these measurements.

\section{References}

1. J. A. Becker and R. O. Nelson, Nucl. Phys. News. 7, 11 (1997).

2. J. Frehaut, NIM 135, (1976), p511.

3. J. Devos, et. al, NIM 135, (1976) p.395.

4. G.J. Schmid, et. al, NIM A 527, (2004), p554.

5. G. F. Knoll, Radiation Detection and Measurements, John Wiley \& Sons, New York, 2000.

6. A. Sayers and C. S. Wu, Rev of Sci. Instr. 28, 10 (1957), p. 758.

7. A. E. Evans, Jr., "Development of High-Pressure ${ }^{3} \mathrm{He}$ Neutron Scintillator Spectrometer," LANL Program Technical Note, LA-Q2TN-82-109. 